\title{
Quiet Geomagnetic Field Representation for All Days and Latitudes
}

\author{
W. H. CAMPBELl ${ }^{1}$, E. R. SCHIFFMACHER ${ }^{2}$, and B. R. ARORA ${ }^{3}$ \\ ${ }^{1}$ U.S. Geological Survey, Mail Stop 968, Box 25046, Denver, Colorado 80225, U.S.A. \\ ${ }^{2} 2155$ Emerald Road, Boulder, Colorado 80304, U.S.A. \\ ${ }^{3}$ Indian Institute of Geomagnetism, Moos Road, Colaba, Bombay, 400005 , India
}

(Received December 16, 1991; Accepted January 6, 1992)

This paper describes a technique for obtaining the quiet-time geomagnetic field variation expected for all days of the year and distribution of latitudes from a limited set of selected quiet days within a year at a discrete set of locations. We used a data set of observatories operated by Indian and USSR scientists in 1976 and 1977 near $75^{\circ} \mathrm{E}$ longitude as illustration. Our method relies upon spatial smoothing of the decomposed spectral components. An evaluation of the fidelity of the resulting model shows correlation coefficients usually above 0.9 at the lower latitudes and near 0.7 at the higher latitudes with variations identified as dependent upon season and field element.

\section{Introduction}

The quiet-time geomagnetic field variations are called " $S q$ " for the solar quiet times that exist when the fields arise. The $S q$ variation has been identified with the currents in the lower ionosphere that are caused principally by the thermal tidal and wind driven motions of charged atmospheric particles across the main field of the Earth. In polar cap regions, the $S q$ currents are not easily separated from the effects of the quiet-time field-aligned current systems. The $S q$ currents are used as field sources for probing the Earth's deep interior. Determinations of $S q$ are required for preparation of the valuable geomagnetic activity indices $K p, A p$, and $D s t$. Estimated $S q$ levels form the baselines about which disturbance fields are measured. Techniques for removal of the $S q$ have been found to greatly improve regional magnetic surveys of interest in crustal composition determinations of the Earth.

In this paper we describe in detail the method we use to establish the daily representations of the year's $S q$ field values over a broad distribution of latitudes. We provide a measure of the accuracy in modeling the original quiet-day records as a function of latitude and season. Three general utility programs, necessary for the representation, are included as appendices: (a) spectral analysis of field changes with significant linear trends, (b) determination of daynumber for a given year, and (c) geomagnetic station coordinate computation.

\section{Selection of the Year's Quiet Days}

Quiet times can be selected in a number of ways. Most methods use the global geomagnetic activity index $K p$ as a gatekeeper. With respect to $K p$, the five quietest days of each month are published regularly in the Journal of Geophysical Research, Planetary and 
Space Science issues. Although this list is adequate for many studies, it produces a quietness level that varies from month to month. Interesting combinations of other activity indices have been used to determine quietness; however, such methods suffered in utility because of the publication delays by production managers for all except the $K p$ index.

Selection of quiet-field times should be a pragmatic balance between the needs for low geomagnetic activity, for sufficient number of days to obtain the yearly pattern of change at an observatory, and for a consistent definition over time. It is known that the $K p$ is a 3-hr range-

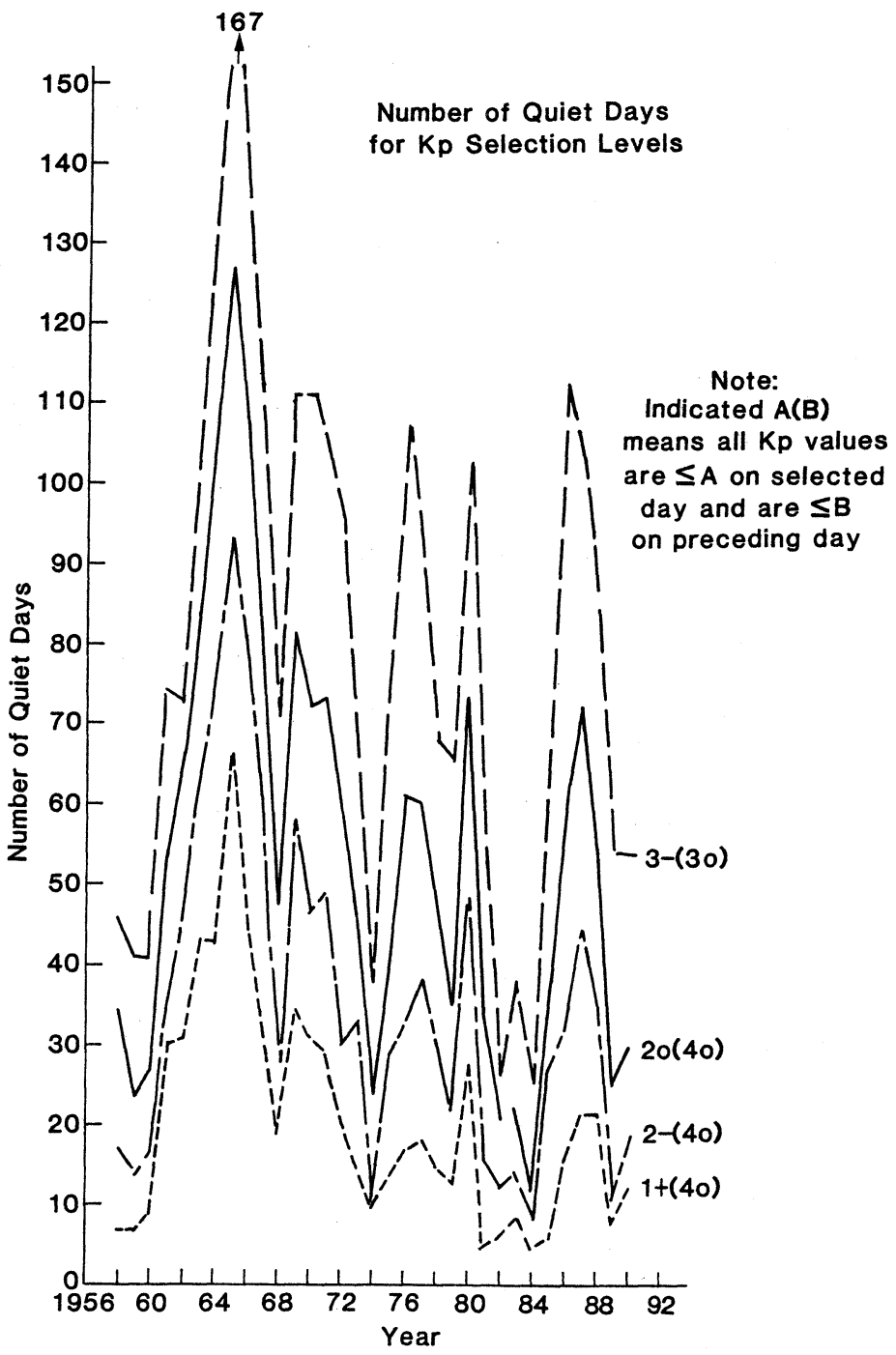

Fig. 1. The number of the quiet days for each year 1956 through 1990. Each trace gives a different selection of quietness defined by values of the 3-hr planetary magnetic activity index, $K p$. Values indicated as $A(B)$ mean that all $8 K p$ values in the UT day were less than or equal to the $K p$ value of $A$ and that all $8 K p$ values of the preceding UT day were less than or equal to the $K p$ value of $B$. 
of-disturbance index that does not respond to the recovery phase of magnetic storms (a decay of the ring current usually lasting less than 24 hours) and shows low values at such times that are still active in the magnetosphere. To avoid such false $K p$ indications of quiet conditions, the values of the preceding day can be included in the quiet-day selection process to verify that no large storm main phases have occurred. Figure 1 is a graph of the yearly number of quiet days in the period from 1958 through 1990 when digitized records are available at World Data Center $\mathrm{A}$. The illustrated selections, shown as $A(B)$, were for quietness determined by all eight $K p$ indices in a UT defined day remaining at or below some level $A$ as well as all eight $K p$ indices on the preceding day remaining at or below some defined level $B$. For example, the least quiet selection illustrated in the figure is $3_{-}\left(3_{0}\right)$ for $K p$ on the day $\leq 3_{-}$and $K p$ on the preceding day $\leq 3_{0}$. The 11-year solar cycle effect is clearly evident in the figure. The 1965 sunspot minimum year stands out as the best quiet year. Our selection of suitable levels was $20\left(4_{0}\right)$ that provided a minimum of 13 days in 1984 and a maximum of 127 days in 1965. Probably because $K p$ is not derived from a uniform global distribution of observatories, occasionally there occur some observatory records of these selected days that are obviously still rather disturbed and need to be excluded from the quiet data set; the technique for identifying such records will be discussed later.

\section{Fourier Decomposition of $S q$}

Our method for estimation of the yearly change in $S q$ uses the separated Fourier spectral components of the individual quiet-day $H, D$, and $Z$ elements of field at a station. The yearly variation of these components is found by a second Fourier analysis that gives a linear trend and set of harmonic coefficients for each original spectral component. For a latitudinal chain of observatories, spatial smoothing techniques provide our best estimate of each element in the $S q$ composition.

Typically the geomagnetic records showed a component of linear trend through the day and through the year. The principal contributor to the daily trend was considered to be either from the quiet-time magnetospheric tail, ring, and field-aligned currents or the boundary currents responding to the deformation of the magnetosphere by the quiet-period flow of solar wind. The secular change of the Earth's main field was thought to be the principal cause of the baseline trend through the year. Modification of the ionospheric $S q$ currents occur because of the seasonal and year-to-year changes in ionization and thermospheric winds; these provide linear and harmonic variations to the Fourier spectral components of the $S q$.

To accommodate the existence of linear trend within the records, an iterative spectral analysis method was devised that proved particularly useful for situations in which there was a small number of regularly spaced data points per wavelength (e.g. with hourly value $S q$ records or with yearly variation data sets). The approach was designed to overcome the harmonic contribution (by the sine terms) to an apparent linear trend by using an iterative method that successively removed those sine contributions from the data sample. With the final spectral values removed from the original data set, a linear fit to the residuals gave the linear trend and data offset (baseline level). The program coding is given in Appendix A. Figure 2 is an example of the application of the program to a sample observatory record. The asterisks are the observatory data points. The bottom section shows the fit of the analysis with 8 Fourier harmonics. The top section shows the decomposition of the program's linear trend and offset determination from the Fourier terms. Because the source of the daily trend and offset was not 
considered to be of $S q$ source origin, these values are removed from further analysis.

The 24-, 12-, 8-, and 6-hr spectral components (1st to 4th harmonic) dominate the $S q$ spectrum at observatories. There are unlikely to be important $S q$ contributions periods shorter than $3 \mathrm{hrs}$ (8th harmonic). For stations with only hourly value records, we consider 6 Fourier harmonics (4-hr component) to be at the limit of resolution. For one-minute-value records, we have carried the analysis to the 12 th harmonic but consider the components below the 8 th to be valueless.

The Indian hourly value data sets that serve to illustrate this article were spectral analyzed

\section{$11 / 16 / 89 \quad H$-COMPONENT CTA}

SLOPE OF LINEAR TREND $=0.007$ GAMMA/MIN. Y-INTERCEPT $=-5.3$ GAMMA
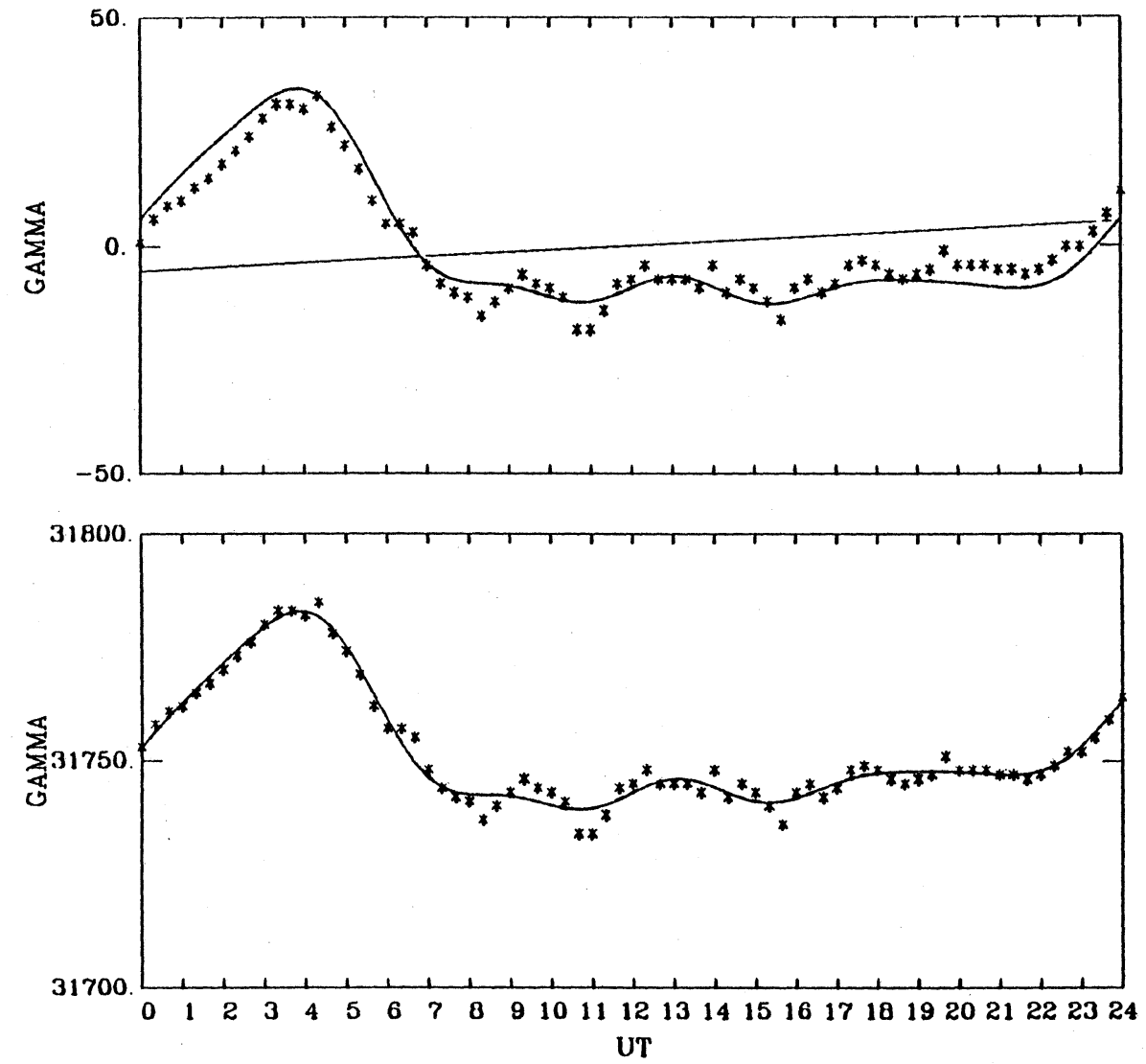

Fig. 2. Spectral component fitting of daily variation field with linear trend. Amplitudes of an example $H$-field element (shown as *'s) are displayed for 20-min intervals of the UT day. The bottom section shows the fit of the eight Fourier analysis harmonics (plus the trend) using the program described in Appendix A. The upper section illustrates the decomposition of the Fourier components and the computed linear trend. The slope of the linear trend in the upper section is $0.4375 \mathrm{gamma} / \mathrm{hr}$; it intercepts the amplitude axis at -5.3 gamma. The mid-point of the trend line, also the zero-level of the Fourier components, in this upper section is at a baseline level of 31752.0 gamma. One-minute field values were used in the analysis. 


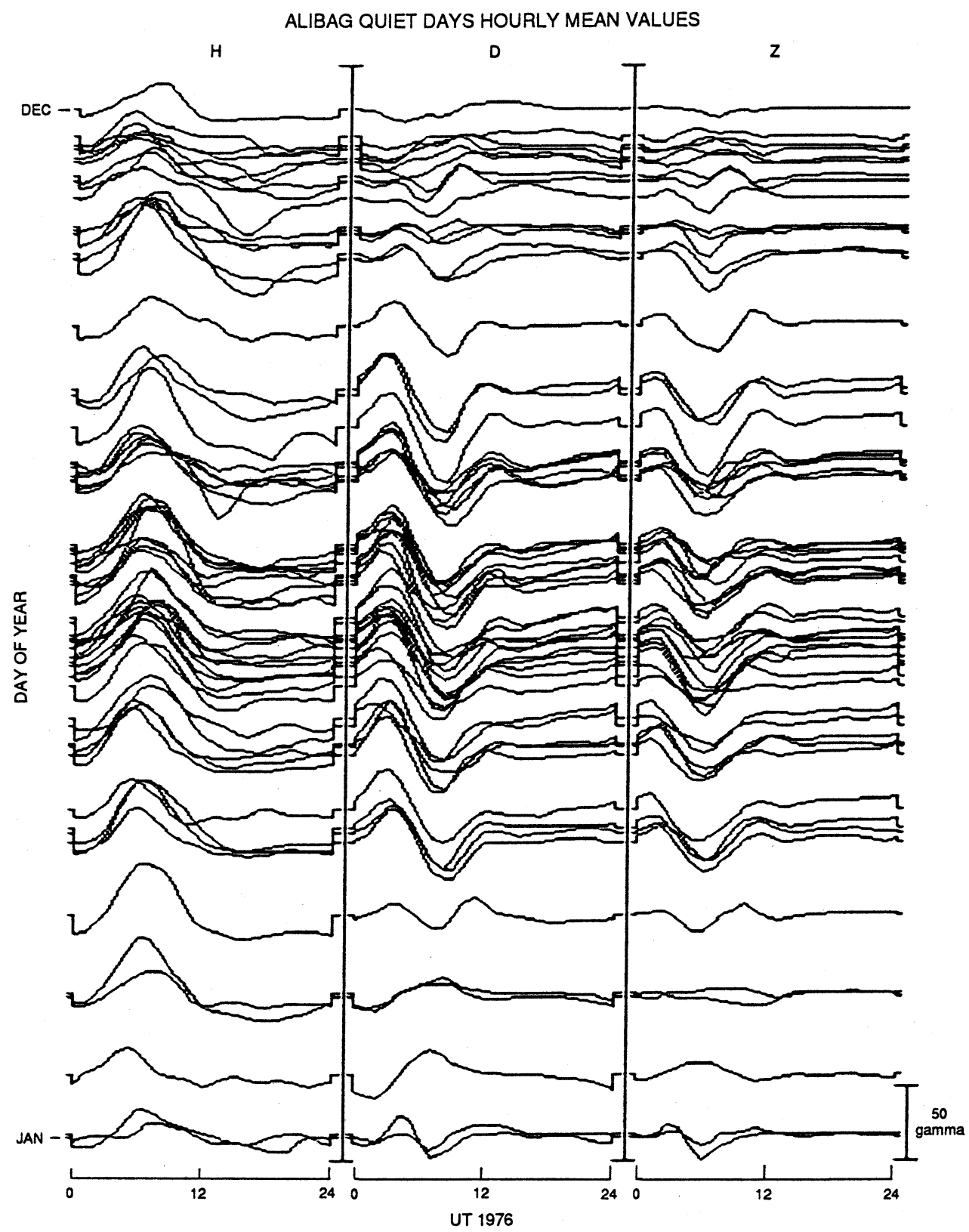

Fig. 3. Geomagnetic daily variations of the field for quiet days at the Alibag observatory (near Bombay, India). Hourly mean amplitude values (gamma scale is shown at lower right) of the $H, D$, and $Z$ elements are given for Universal Time (UT) hours. Records are offset in this display to indicate the day of the year 1976 from January 1 (bottom JAN) to December 31 (top DEC). The beginning and end of each trace returns to the daily mean level at that date. 


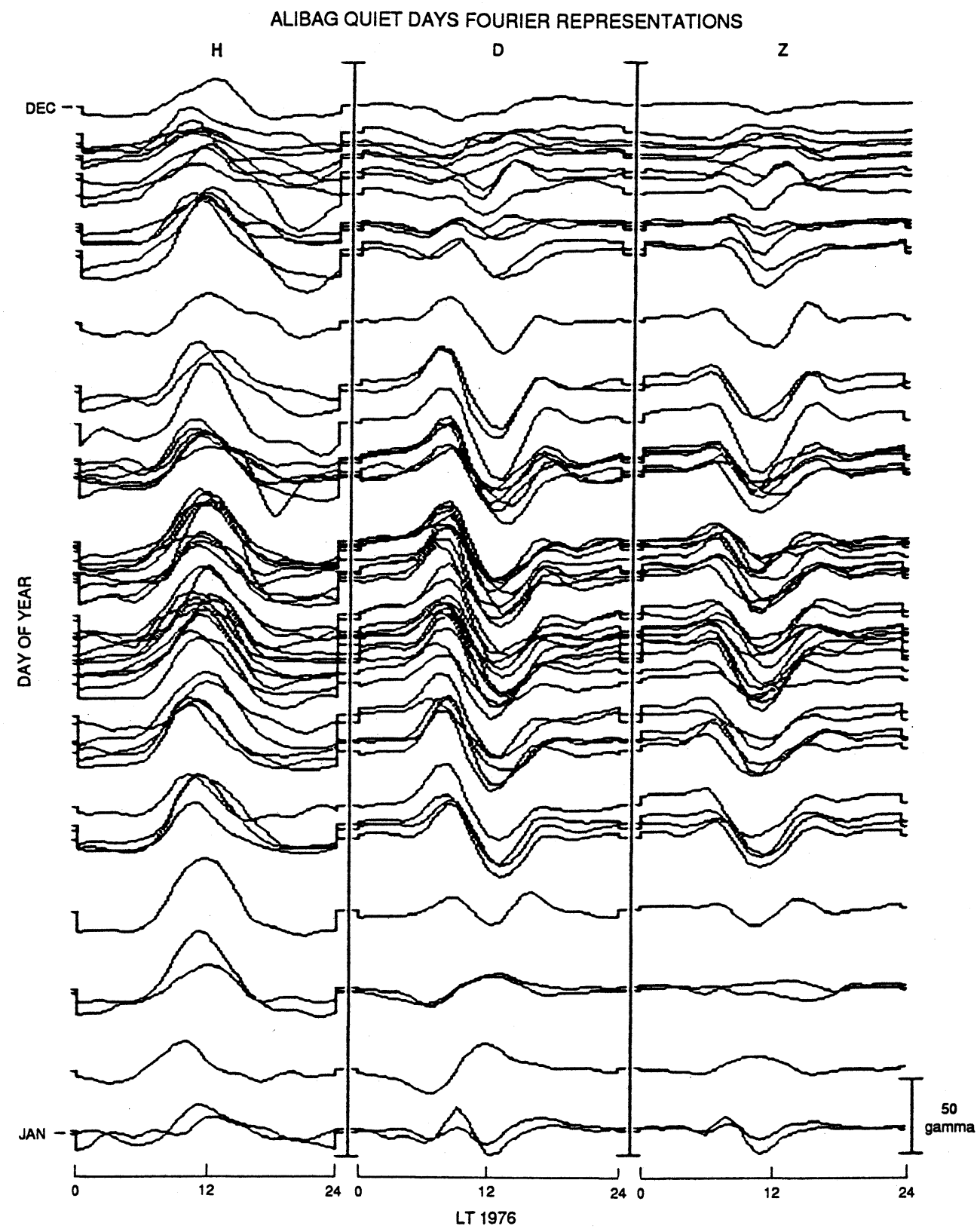

Fig. 4. Spectral representations of the field for quiet days at the Alibag observatory. Hourly values (gamma scale shown at lower right) of the $H, D$, and $Z$ elements are given for Local Time (LT) hours. Records are offset in this display to indicate the day of the year, 1976, from January 1 (bottom JAN) to December 31 (top DEC). The beginning and end of each trace returns to the daily mean level at that date. 
to the 6th harmonic. The spectral components were phase shifted by the station longitude to represent the equivalent Fourier coefficients in local time. Figure 3 shows the geomagnetic daily variations of the field for quiet days of 1976 at the Alibag observatory south of Bombay, India. Figure 4 shows, for the same days, that the reconstruction of the fields from the spectral representations in local time is essentially indistinguishable from the raw data.

\section{Annual Variation}

Each of the cosine and sine spectral amplitude components from a given observatory is considered separately to determine its variation through the year (see Appendix B for a convenient program to determine the day-number for a given year). In our study, both 1- and 2-yr samples were evaluated to see if there was any improvement in representation with the larger samples. It was found that a 1-yr distribution of data was adequate for general studies, but the 2-yr sampling was necessary for subsets with fewer days such as the $S q$ days selected by IMF sectors. Wild coefficient values, determined to be those greater than 2.5 standard deviations away from the average of the four adjacent values, were excluded from the analysis, replaced by the mean of the adjacent two values, and relocated midway between them. When there were more than seven occasions of exclusion on a identical day, the date was assumed to be a BADDAY for the regional $S q$ studies and excluded as if it were an active day with high $K p$.

We used the same Fourier-analysis-with-linear-trend program described above for the 4harmonic seasonal change analysis (and 2-yr component when the larger data set was included). The program requires an evenly spaced data set. Usually a running parabolic fit to data points is used to determine data values at evenly spaced time intervals. The very irregular, seasonally grouped spacing of the quiet days often produced wild results from this parabolic method. Instead, we simply connected a straight line between points and extrapolated to the evenly spaced time location.

Figure 5 is an example of a 2 -yr analysis performed on the 24-hr cosine ( $A 1)$ and sine $(B 1)$ Fourier amplitude coefficients. The $A 1$ and $B 1$ representations show the seasonal change with and without linear trend and the composite fit to the data points. It is from these latter curves, for each of the Fourier coefficients $A 1$ to $A 6$ and $B 1$ to $B 6$, that the quiet-day values for each day of the year are reconstructed. Figure 6 illustrates Alibag quiet-day variation for every 10 days of 1976 using the 2-yr analysis method. Note the low-latitude equinoxial effects in $H$ amplitudes and the summer maxima in $D$ and $Z$.

\section{Latitude Smoothing}

At times when data from a number of observatories are available along or near a given meridian, it is convenient to determine the latitude variation of the $S q$. Such a set was gathered by the Indian Institute of Geomagnetism, in cooperation with USSR colleagues, for geographic longitudes near $75^{\circ} \mathrm{E}$. longitude. Figure 7 illustrates the location of these stations. The dashed lines show the geomagnetic longitudes in $15^{\circ}$ steps about the Trivandrum (TRD) observatory location and $15^{\circ}$ latitude steps about the geomagnetic equator. See Appendix $\mathrm{C}$ for a convenient program to determine geomagnetic latitudes of observatory locations.

A special technique is used to smooth the coefficients for the year's $S q$ at each station so 

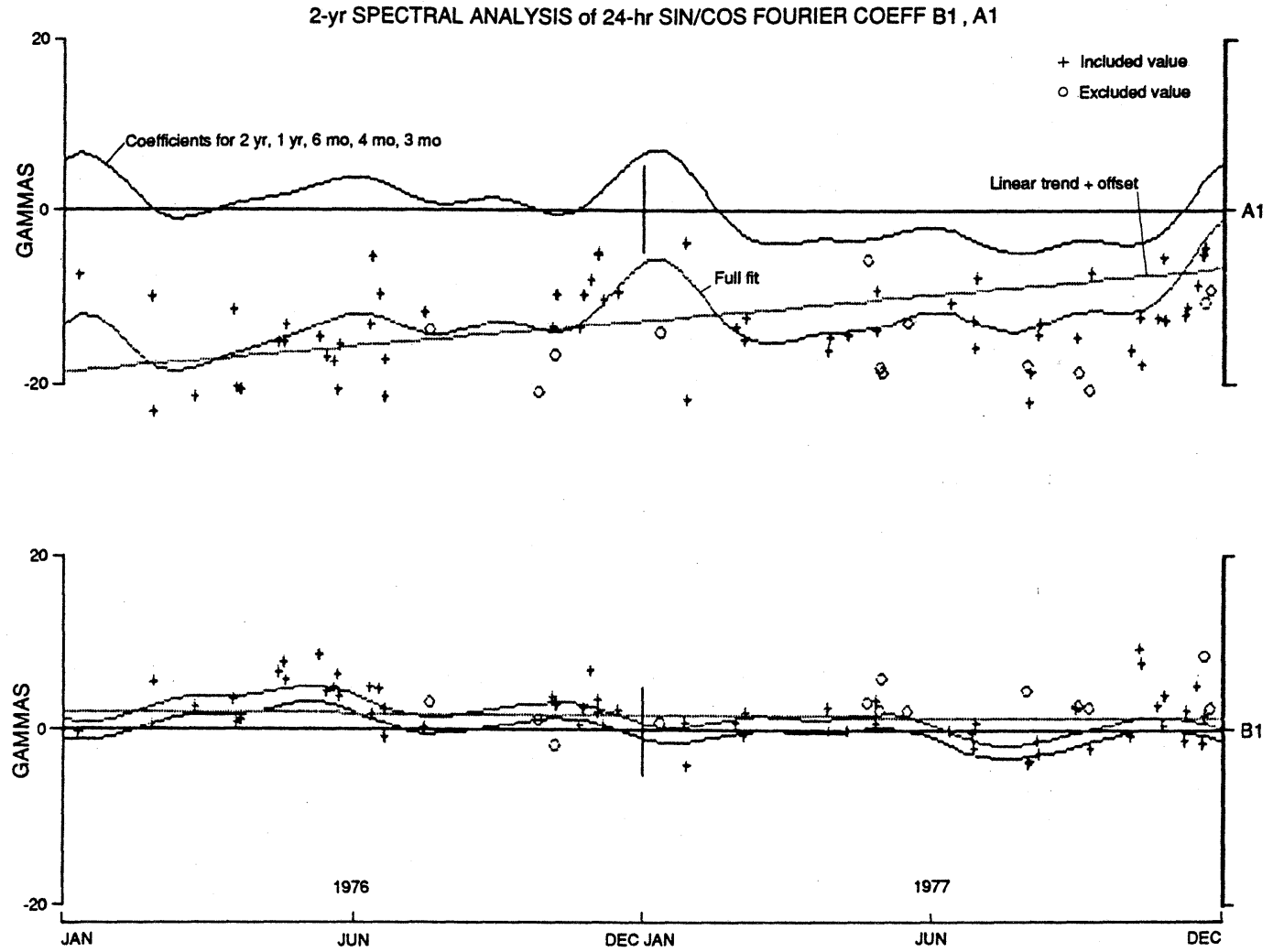

Fig. 5. Spectral analysis of the 24-hr Fourier cosine and sine coefficients, $A 1$ and $B 1$, for 1976 and 1977 containing the 2-yr, 1-yr, 6-mo, 4-mo, and 3-mo components. The Alibag observatory values of amplitude (in gamma) are plotted versus day number in the year. Top section is for the $24-\mathrm{hr}$ cosine coefficient, $A 1$; bottom section is for the $24-\mathrm{hr}$ sine coefficient, $B 1$. The +'s represent the values determined in the individual day Fourier analysis. The $\bigcirc$ 's represent values excluded for reasons given in the accompanying text. In the upper section, the upper smooth curve shows the seasonal spectral representation without the computed linear trends; the lower smooth curve shows the linear trend, offset, and full fit of the spectral analysis to the $A 1$ values. In the lower section, the lower smooth curve shows the spectral representation without the computed linear trends; the upper smooth curve shows the linear trend, offset and full fit of the spectral analysis to the $B 1$ values. 
ALIBAG Sq for YEAR from set 76F20.ANN

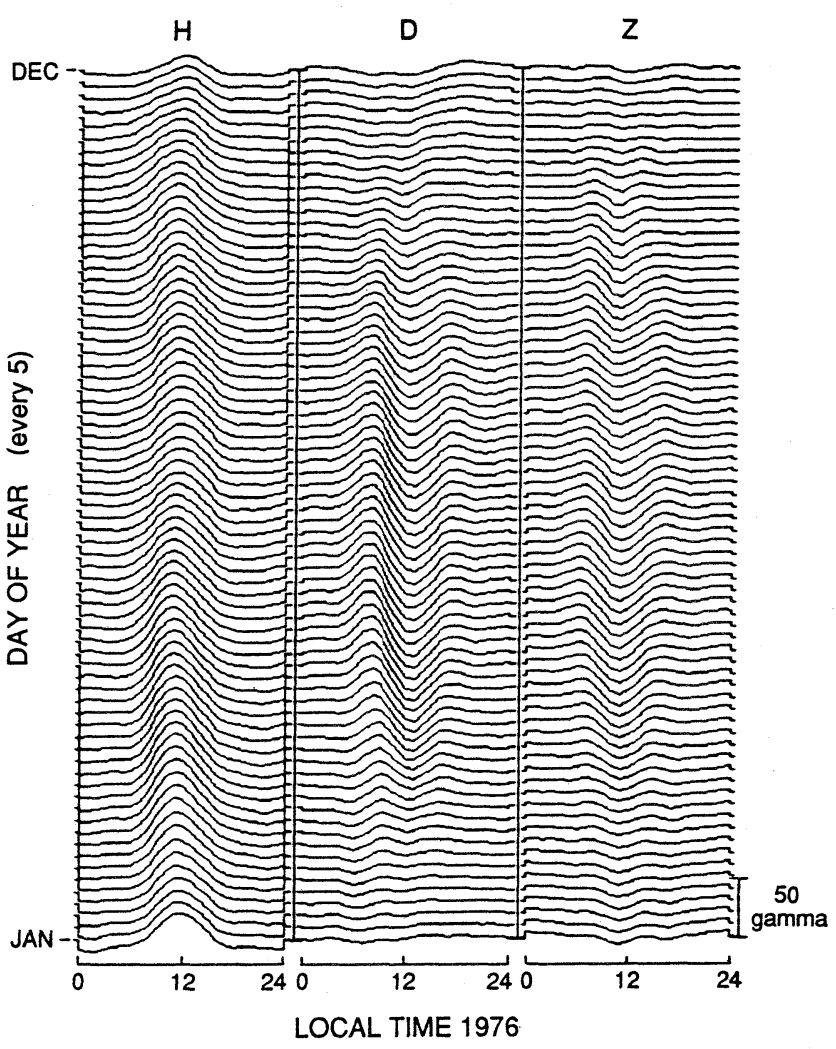

Fig. 6. Full-year representation of the Alibag quiet-day field amplitude variations for $H, D$, and $Z$ elements (gamma scale at lower right) shown in Local Time (LT). Records are offset in this display to indicate the day of the year 1976 from January 1 (bottom JAN) to December 31 (top DEC). The beginning and end of each trace returns to the daily mean level at that date.

that a representative field may be described at any latitude location along the selected meridian. A compromise solution needed to be found for five conflicting spatial definition problems: (a) local time (geographic location) largely governs the organization of source region ionization as well as the thermal tidal and wind patterns that drive the ionization; (b) the geomagnetic coordinates of the main field are important for defining the magnitude of the dynamo process in the ionosphere; (c) a uniform, Earth-centered coordinate system is needed for simple mapping of the source current and for the determination of conductivity profiles within the Earth; (d) near the equator, the magnetic dip latitude, which can vary greatly from the dipole latitude, organizes the dynamo current patterns; (e) in the polar regions, the observed ionospheric currents are best represented by the "eccentric axis dipole" or the "corrected dipole" coordinate system.

The local time at each station was taken to be the organizing feature in longitude and the geomagnetic coordinates were assumed to give the dominant latitude arrangement. The three most equatorial stations were reassigned their dip latitude as pseudo geomagnetic latitude 


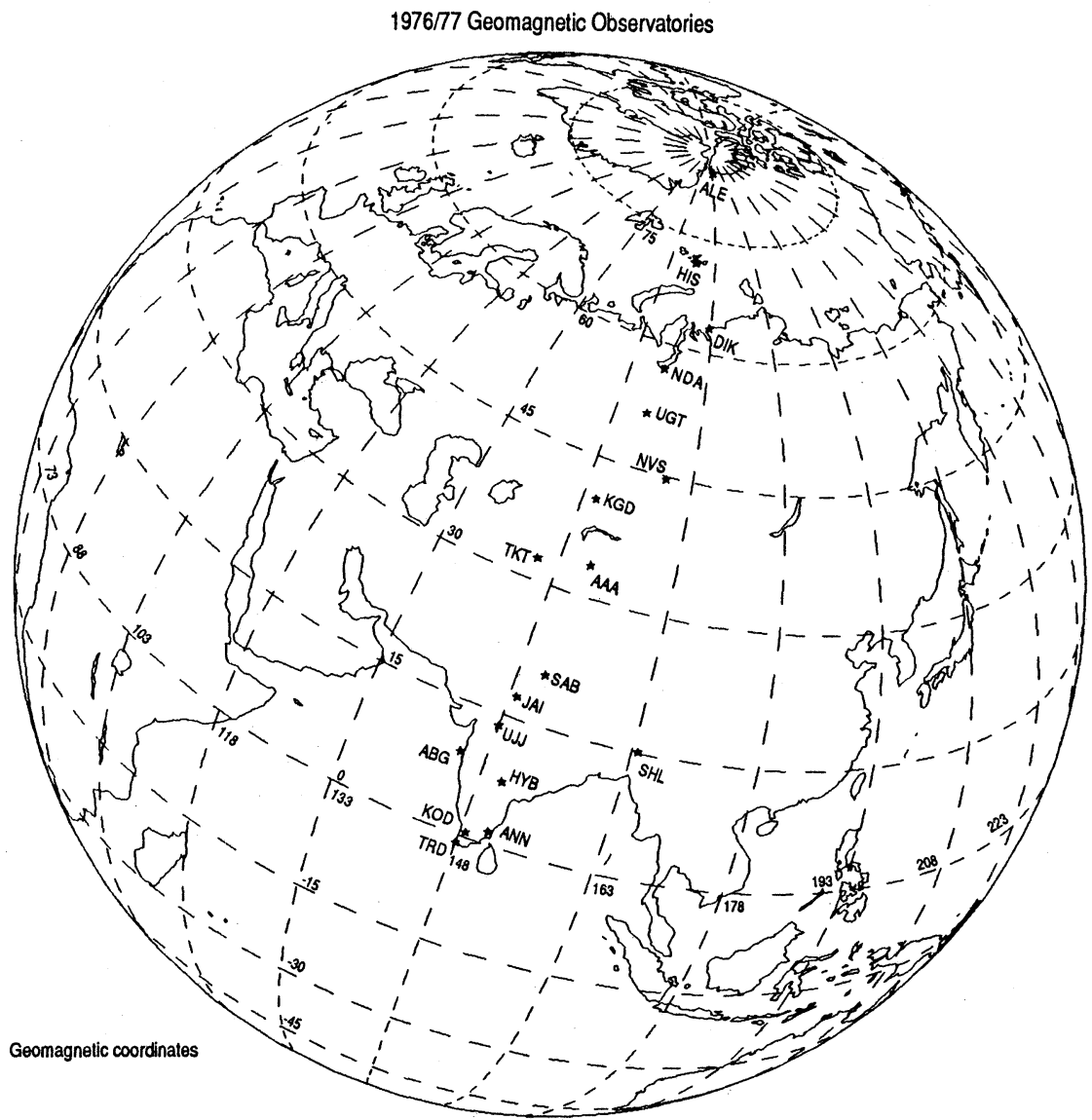

Fig. 7. Location of the $1976 / 1977$ observatories used in the latitude smoothing described in the text. The dashed lines show the geomagnetic longitudes in $15^{\circ}$ steps about the Trivandrum (TRD) observatory location and $15^{\circ}$ latitude steps about the geomagnetic equator.

$\left(0.00^{\circ}\right.$ for $\mathrm{TRD}, 1.42^{\circ}$ for $\mathrm{KOD}$, and $2.30^{\circ}$ for $\left.\mathrm{ANN}\right)$. For smoothing purposes, values from stations within, and the first one just above, $5^{\circ}$ of the equator were mirrored into the opposite hemisphere and values from stations within, and one just south of, $5^{\circ}$ from the pole were mirrored as if they were above $90^{\circ}$.

Latitude smoothing of each separate coefficient was then carried out in the following way. A linear interpolation of the particular coefficient was determined between observatory latitude locations. Starting at $5^{\circ}$ south of the equator and using a $10^{\circ}$ latitude window, values were read at every $0.5^{\circ}$ interval. To these points, a second-order polynomial fit was determined, and the polynomial-fit value was assigned to the window-center location. The window was then moved $2^{\circ}$ northward and the process repeated until values were assigned to every $2^{\circ}$ from the equator to the pole. Figure 8 shows the fitting of the $H$ field element $24-\mathrm{hr} A$ cosine spectral component's yearly change as Fourier 1-yr $A 2$ (cosine) and $B 2$ (sine) spectral component values. 
1976/77 H 24-hr A cos FOURIER COEFFICIENTS

1-yr A2 cos \& B2 sin Spectral Components

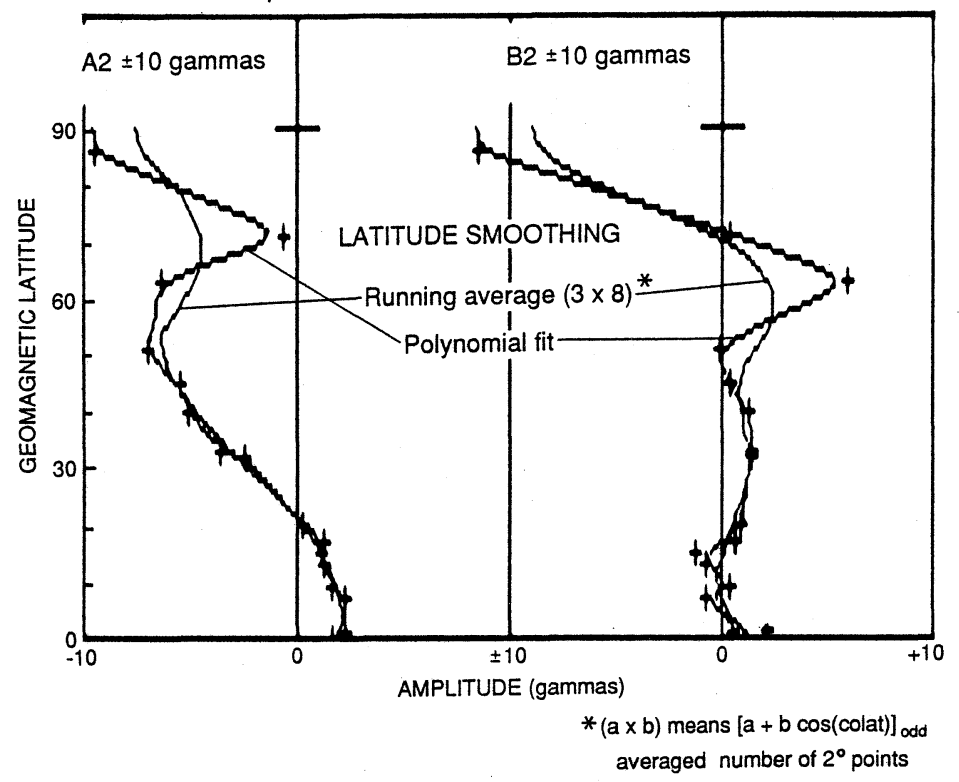

Fig. 8. Polynomial and running-average fit to $H$ field element, 24-hr cosine, 1-yr cosine $(A 2)$ spectral component (left section) and sine (B2) spectral component. Latitude scale for the contributing observatory values (shown as +'s) is given at left. Amplitude in the horizontal direction is scaled at bottom in gammas. The running-average window was 3 -steps $\left(2^{\circ}\right.$ each step) wide at the equator and $3+8=11$ steps wide at the pole; this window increased in odd-numbered steps as the cosine of the geomagnetic colatitude (see text).

These station values are indicated as +'s; the $2^{\circ}$ polynomial-fit values are indicated as a smooth curve.

Next a running variable-window smoothing was applied to the polynomial-fit values. This window was 3 of the $2^{\circ}$ values wide starting at the equator and $3+8=11$ values wide at the pole. The polynomial-fit values at $0^{\circ}$ and $2^{\circ}$ were made to be unchanged by the smoothing; values in the polar region were appropriately mirrored to allow smoothing to $90^{\circ}$ latitude. The variable window width was adjusted to increase in odd-numbered steps accommodating, as nearly as possible, a cosine of colatitude change. The average of the values within the window were assigned to the window midpoint (except near the equator, as noted above). Figure 8 shows the effect of this running-average smoothing on the station values. Note the increasing variability of the fitting with increasing latitude that will be evaluated below.

\section{Data Representation}

The file of $2^{\circ}$ coefficients obtained from the variable smoothing described above allows, by extrapolation, the re-creation of an estimated quiet-field variation at any latitude near the $75^{\circ} \mathrm{E}$. geographic longitude. Figure 9 illustrates the quiet-field daily variation in $H, D$, and $Z$ for each $2^{\circ}$ of latitude obtained from the variable smoothing described above. 
To provide some measure of the fitting of the model as a function of field element, latitude location, and season, we made a comparison of the Fourier harmonic portrayal of the original $S q$ field to the smoothed latitude representation. The correlation coefficient for the 144 tenminute values of field from the two models, averaged for the originally available days in the selected 3-month seasonal groups, was determined as a function of latitude. For the same samples and grouping, we also calculated the magnitude of the difference between the amplitudes of the two field representations and found the average difference magnitude as a percentage of the range of the Fourier harmonic representation. These two measures of the fit are shown as a function of latitude in Fig. 10.

Two observatory locations were found to be particularly unusual in their fitting: Shillong (SHL at $15.1^{\circ}$ ) and Nyda (NDA at $56.9^{\circ}$ ). The Shillong observatory was about one hour east of the other station longitude locations and out of line with the data trend followed by the nearby observatories. A review of the original Nyda records indicated that this observatory was probably not carefully maintained during the observation period. The latitude smoothing of records was redone removing the two troublesome sites. In Fig. 10, the comparison shows, with

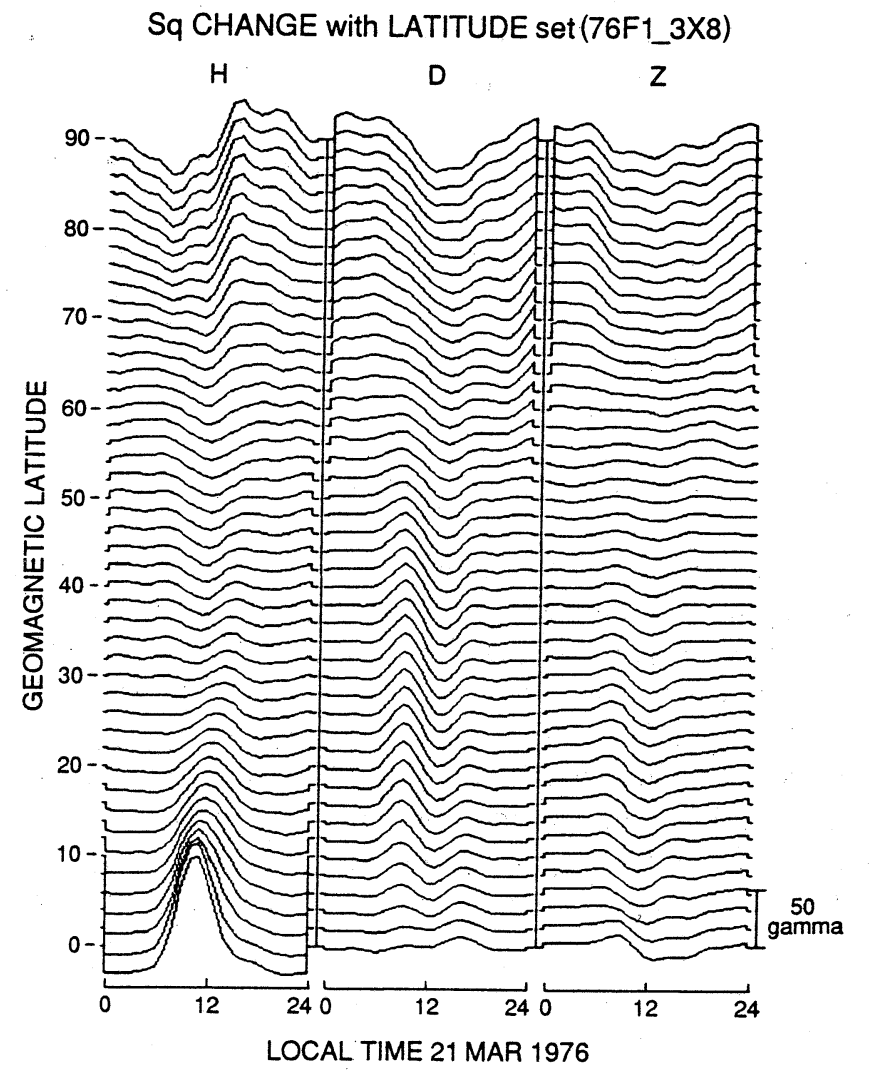

Fig. 9. Amplitudes of the quiet field daily variation in $H, D$, and $Z$ elements (scale given at bottom right) displayed for each $2^{\circ}$ latitude from the equator (bottom traces) to the pole (top traces). Values, given in local time for 21 March 1976 (horizontal scales), were obtained from the latitude smoothing technique described in the text. The set name, $76 \mathrm{~F} 1 \_3 \times 8$, is a coded file identification. 


\section{EFFECT OF LATITUDE SMOOTHING OF QUIET DAY RECORDS} $\left(75^{\circ} \mathrm{E}\right.$ DATA SET 1976)
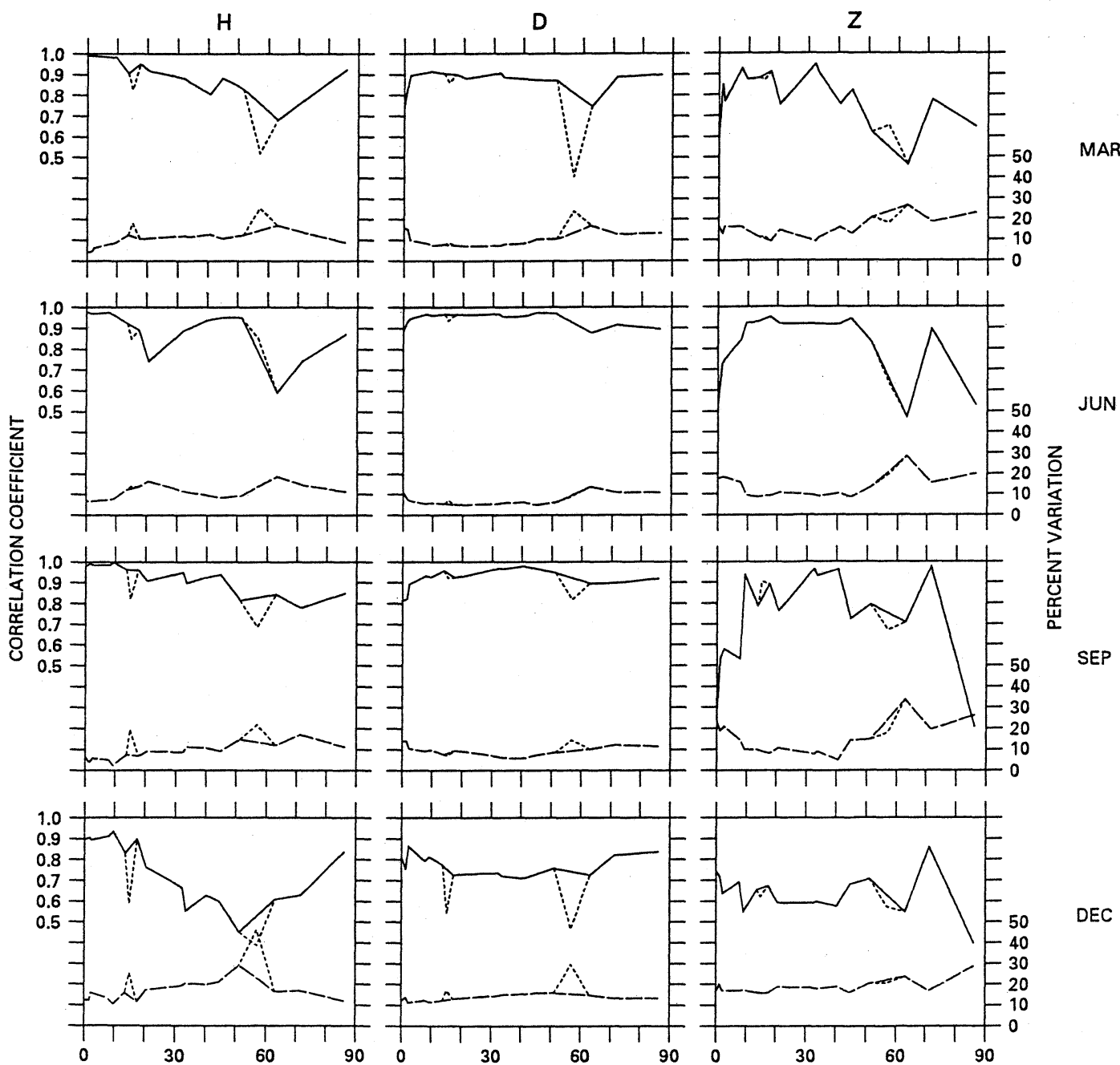

Fig. 10. Correlation coefficients and percent variation obtained in a comparison of the original Fourier representation of the data to the reconstructed values from the latitude smoothing technique described in the text. Values for the three $H, D$, and $Z$ elements and 3-month seasons centered on March, June, September, and December are displayed separately as a function of the observatory latitudes (scales at bottom). Dashed curves are the correlation coefficients (scales to left); solid curves are the percent variation (scales to right). Observatory contributions at $15.1^{\circ}$ and $56.9^{\circ}$ were excluded from the smoothing, but evaluations of the fit at these locations are illustrated to justify their removal. 
dashed lines, how data from these two sites typically have lower correlation coefficients and higher percent variation in amplitude from that expected of the regional behavior by the observatories at nearby latitudes. The discrepancies appeared largest on the $H$ and $D$ field elements and least on the $Z$ element.

Figure 10 illustrates some general behaviors of the fitting with element, latitude, and season. The $D$ component generally fit best of all the elements because of its consistent daily amplitude pattern at about all latitudes and seasons. In unique regions and for special times when one field element was at low amplitude compared to the other two elements, the correlation coefficient was low and the variation value high; e.g. consider the $Z$ element near the equator in March, June, and September. Occasionally at the $S q$ source current latitudes near $20^{\circ}$, where the $H$ component is small, we also see low correlation and high variation values (e.g. the June record). Usually, the model fitting degraded at the higher latitudes; irregular auroralzone and polar-cap activity could account for this feature. In general, the correlation coefficient between the observatory fields and the smooth, representation was better than 0.9 at low latitudes and about 0.7 at the higher latitudes; the corresponding percent variations were about 10 percent and 20 percent.

We wish to thank the Indian Institute of Geomagnetism (Bombay, India), the World Data Center A for Solar Terrestrial Physics (Boulder, Colorado, USA), and the World Data Center B2 (Moscow, USSR) for their help in providing the primary data for this study. The work was made possible by a grant from the U.S.-India Special Rupee Fund project USIF-USGS 142 administered by the U.S. State Department and the Indian Department of Science and Technology. 


\section{APPENDIX A}

Computer program for Fourier spectral analysis of evenly spaced observatory data samples with embedded linear trend.

'A:FOURXX.BAS in a form of BASIC language; Program by พ.H.C.

'Fourier analysis with linear trend removal

'Iterations to remove contributions of sine terms to linear part

' For application to study of geomagnetic daily variations

'Need evenly spaced sample points with values at beginning end

DEFDBL A-Z 'All tagless varibles will have double precision DEFINT I-N 'Except Integer for varibles begining $I$ to $N$

INPUT "File name ", F\$ 'Give H-, D-, or Z-element file name N9=15 'INPUT "Minutes between samples", N9' TEST for N9=15 $\mathrm{K} 1=12$ 'INPUT "Total Fourier harmonics wanted", $\mathrm{K} 1$ ' TEST for $\mathrm{K} 1=12$ I9=6 'INPUT"Max number of iterations for fitting ", I9 'Use 5 to 8 $\mathrm{N} 8=1440 / \mathrm{N} 9$ ' N8 Data intervals and $\mathrm{N} 8+1$ data points

DIM $Y(N 8+1), Y O(N 8+1), X(K 1), A(K 1), B(K 1)$ 'A cos \& B sin

LPRINT "Start ";DATES;" ";TIMES:LPRINT

LPRINT "Fourier Analysis of ";FS;

IPRINT " file with ";N9;" min/samples for ";K1;" harmonics"

LPRINT " $A(\mathrm{~K})$ and $B(\mathrm{~K})$ coeff. for $24 / \mathrm{K} \mathrm{hr}$ comp.; $\mathrm{K}=1$ to $\mathrm{K} 1$ "

LPRINT "Uses ";N8+1;" pts with values at beg. and end of the day"

$\mathrm{PI}=3.141592653589793$ 'Note: degrees need be converted to radians $Y(0)=0$ 'Note: OPTION BASE 0 so no value is used at $Y(0)$

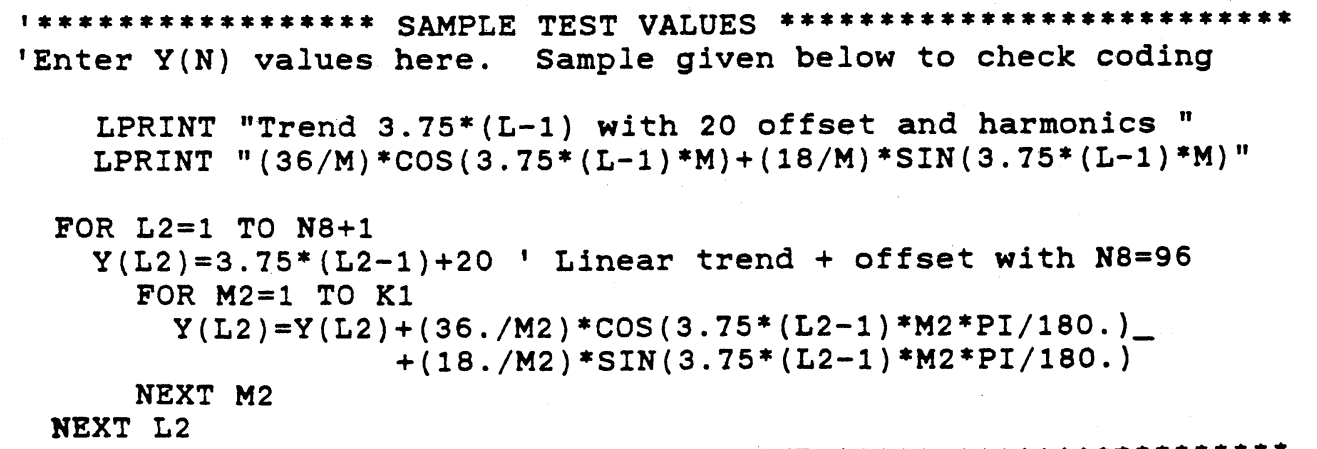

$1 * * * * * * * * * * * * * * * * *$ END SAMPLE TEST INPUT

FOR $M=1$ TO $N 8+1$ ' Save values for linear fit of difference.

NEXT M $Y O(M)=Y(M)$

LPRINT : LPRINT : LPRINT " ";FS;" ";K1;"-term harmonic analysis"

$X(1)=0: X(2)=0: X(3)=0: X(4)=0$ ' Initialize values 


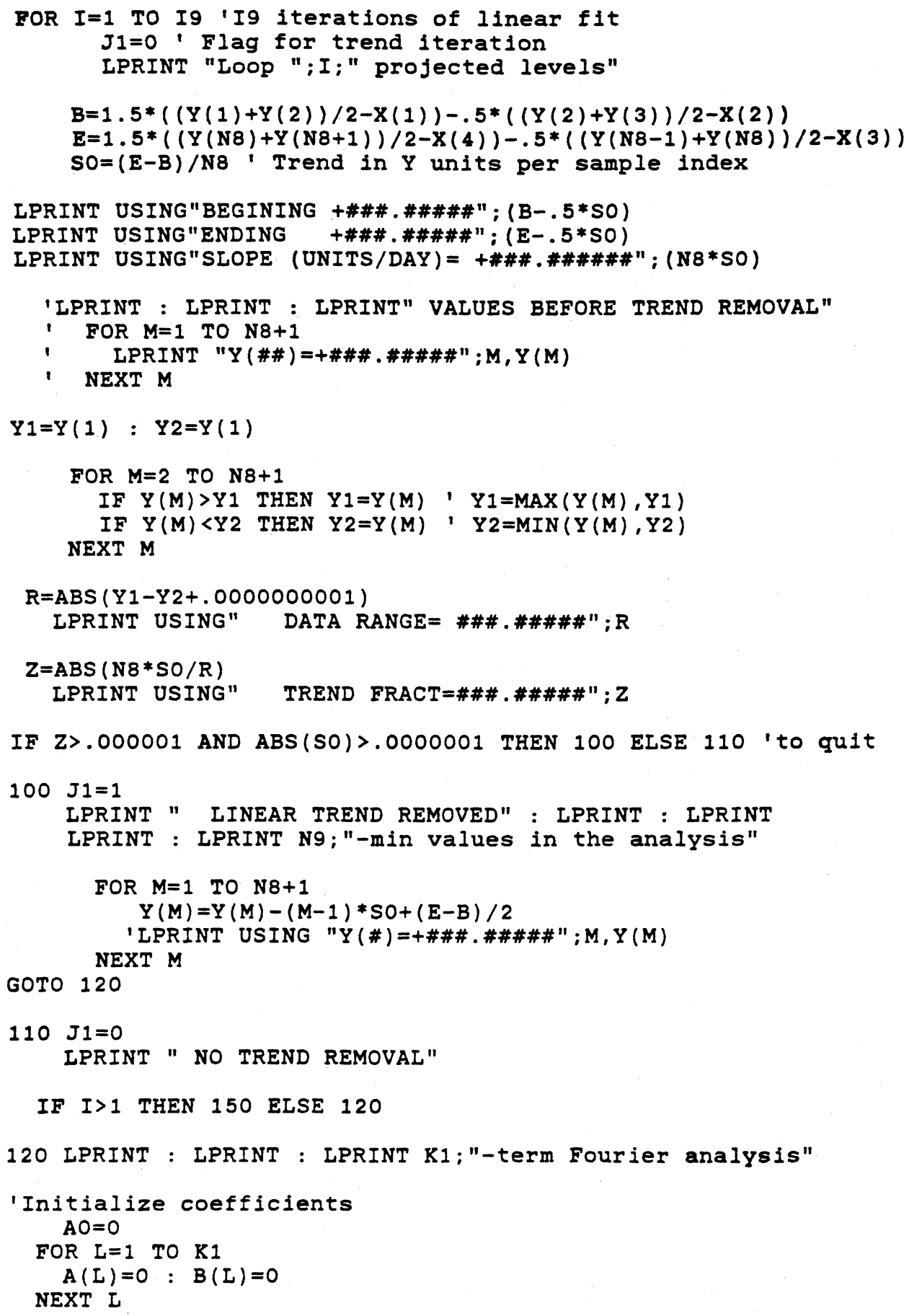


FOR $M=1$ TO N8

$T 1=M * N 9 / 4-N 9 / 8$

$11.25 \mathrm{deg}$ for $5-\mathrm{min}$ sample; 0.625 to shift to mid sample location

$A O=A O+(Y(M)+Y(M+1)) / N 8$

FOR $\mathrm{K}=1$ TO $\mathrm{K} 1$

$A(K)=A(K)+(Y(M)+Y(M+1)) * \operatorname{COS}((P I / 180) *.(K * T 1)) / N 8$

NEXT $K$

$B(K)=B(K)+(Y(M)+Y(M+1)) * S I N((P I / 180) *.(K * T 1)) / N 8$

NEXT $M$

IPRINT USING" $\quad(A O / 2)+\# \# \#$ \#\#\#\#";AO/2 : IPRINT

FOR $\mathrm{K}=1$ TO $\mathrm{KI}$

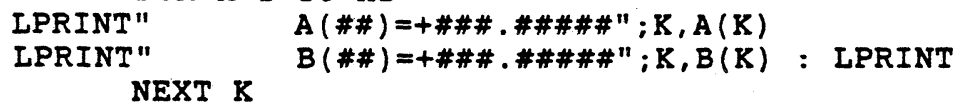

NEXT K

IF $J 1=1$ THEN 130 ELSE 150

130 IF I=I9 THEN 150 ELSE 140 'Change I for more iterations

140

$X(1)=0: X(2)=0: X(3)=0: X(4)=0$ 'Initialize

FOR $\mathrm{K}=1$ TO $\mathrm{K} 1$

$X(1)=X(1)+B(K) * \operatorname{SIN}((P I / 180) *.(K * .5 * N 9 / 4))$

$X(2)=X(2)+B(K) * \operatorname{SIN}((P I / 180) *.(K * 1.5 * N 9 / 4))$

$X(3)=X(3)+B(K) * \operatorname{SIN}((P I / 180) *.(K *(N 8-1.5) * N 9 / 4))$

NEXT $K$

$X(4)=X(4)+B(K) * \operatorname{SIN}((P I / 180) *.(K *(N 8-.5) * N 9 / 4))$

NEXT I

'Determine regression line of diff. between orig. and final $Y()$. $150 \times 1=0: X 2=0: X 3=0: Y 1=0$ 'Initialize sums of $X, X Y, X-2, Y$

FOR $M=1$ TO N8

$Y O(M)=Y O(M)-Y(M)$ ' Original minus final values.

$\mathrm{X} 1=\mathrm{X} 1+\mathrm{M}$ ' Sum of $\mathrm{X}^{\prime} \mathrm{s}$ i.e. $\mathrm{M}^{\prime} \mathrm{s}$

$X 2=X 2+M * Y O(M)$ ' Sum of $X Y^{\prime} s$

$Y 1=Y 1+Y O(M)$, Sum of $Y^{\prime} S$

NEXT $M$ $X 3=X 3+M^{-2}$, sum of $X^{-1} 2^{\prime} s$

$B=(N 8 * X 2-X 1 * Y 1) /(N 8 * X 3-X 1-2)$

$A=Y 1 / N 8-B^{*}(X 1 / N 8-1)$, For $Y=A+B X$

LPRINT

LPRINT USING"Offset +\#\#\#\#.\#\# plus +\#\#\#.\#\# mid-sample";AO/2+A,-A

LPRINT "For $Y=A+B X$ about mid-sample axis we obtain:"

LPRINT USING " A=+\#\#\#.\#\#\# and $B=+\# \# \#$. \#\#\#"; $A, B$ 
S1=B*N8 ' To pass back as units/day

LPRINT USING "SLOPE $=+\# \# \#$.\#\#\# gamma/day";S1

LPRINT "Coefficients referenced to U.T. day"

FOR $\mathrm{K}=1$ TO $\mathrm{K} 1$ 'Discrete sampling of smooth $\mathrm{sin} / \mathrm{cos}$ curves

$A(K)=A(K) / \operatorname{Cos}(P I * K / N 8)$

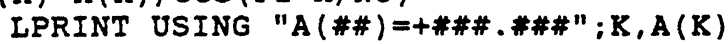

$B(K)=B(K) / \operatorname{COS}(P I * K / N 8)$

NEXT $K$

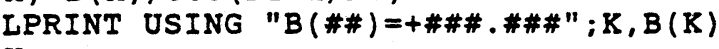

LPRINT:LPRINT "End ";DATES;" ";TIME\$

LPRINT CHRS(12)'Page feed

END

APPENDIX B

Program for determination of the day-number given the day, month, and year.

'DAYNUM.BAS for day-number, D1, in a form of BASIC language

'Given year, month, day, and day-number are integer ( $($ ) variables

'Variables $J 1$ and $J 2$ are double precision integers (\&)

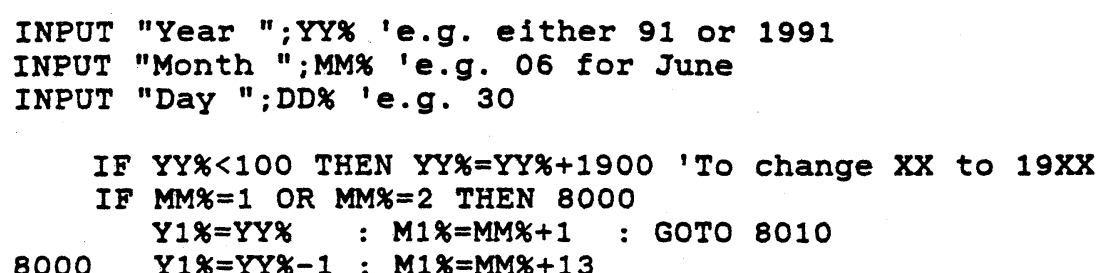

IF $Y Y \%<100$ THEN $Y Y \%=Y Y \%+1900$ 'To change $X X$ to $19 X X$

IF MM\% $=1$ OR MM\% $=2$ THEN 8000

Y1\% $=Y Y \%: M 1 \%=M M \%+1$ : GOTO 8010

$8010 \quad J 1 \&=D D \%-I N T(.75 * I N T(Y 1 \% / 100)-7)$ $+\operatorname{INT}(365.25 * \mathrm{Y} 1 \%)+\operatorname{INT}(30.6 * \mathrm{M} 1 \%)$

$J 2 \&=1-\operatorname{INT}(.75 * \operatorname{INT}((Y Y \%-1) / 100)-7)$

$+\operatorname{INT}(365.25 *(Y Y \%-1))+\operatorname{INT}(30.6 * 14)$

$D 1 \%=J 1 \&-J 2 \&+1 \quad ' D 1$ is integer day number (January $1=1$ )

IPRINT USING "For year \#\#\#\# on day \#\# of month \#\#";YY\%,DD\%, MM\% LPRINT USING " The day-number is \#\#";D1\%

END 
APPENDIX C

Program GMCORD.BAS to find geomagnetic dipole cordinates from geographic locations. Program uses a mathematical procedure suggested by $A$. C. Fraser-Smith, Centered and eccentric geomagnetic dipoles and their poles, 1600-1985, J.Geophys.Res., v. $55,1-16,1987$.

'A:GMCORDA.BAS in a form of BASIC language; program by w.H.C.

' Determine geomag. latitude, longitude and declination

' from geographic latitude, G3, and longitude, G2.

'Model uses extrapolated 5-Yr DGRF/IGRF coefficients.

DEFDBL A-Z 'All tagless varibles will have double precision

$$
P I=3.141592653589793
$$

'DEFine $F N$ for conversions to radians and trignometric relations DEF FNRtoD $(D 2)=D 2 * 180 . / P I$ 'Convert radians to degrees

DEF FNDtOR(D2) = D2*PI/180. 'Convert degrees to radians

DEF FNARCCOSR (D2)=PI/2.-2.*ATN(D2/(1.+SQR(1.-D2*D2))) 'ArCCOS

DEF FNARCSINR (D2) $=2 .{ }^{*} \operatorname{ATN}(D 2 /(1 .+\operatorname{SQR}(1 .-D 2 * D 2)))$ 'ArcSin

DIM $G(11,3)$ 'Dimension Gauss coef. groups of 3 for 11 sets (DATA)

9020 INPUT "Year (1945 to 1995$)=" ;$ YYYY

INPUT " Day-number ="; D1 'See Appendix B program

$Y=Y Y Y Y+D 1 / 365$. 'Date as decimal year

LPRINT USING "Analysis year is \#\#\#\#\#\#"; $Y$

IF $Y=1945$. THEN $Y=1945.001$ 'No models before 1945

IF $Y=1995$. THEN $Y=1994.999$ ' 1995 extrap. from earlier years

IF $Y<1945$. OR $Y>1995$. THEN 9120 ELSE 9500 ' if OK read DATA

9120 PRINT "Year outside model range" : GOTO 9020 'Restart

9130 INPUT "What is station name ", STAS

INPUT "What is station latitude ", G3 'Use + north, - south INPUT "What is station longitude ",G2 'Use + east, - west

$9140 \mathrm{Y} 1=\mathrm{Y}-\mathrm{YO}$ ' Coefficient adjustment increment from extrapolation $G O=G O+Y 1 * G 9$ : $G 1=G 1+Y 1 * G 9$ : $H 1=H 1+Y 1 * H 9$

9150 LPRINT "Gauss coefficients were taken to be: "

LPRINT USING " GO=+\#\#\#\#.\#";GO

LPRINT USING " G1=+\#\#\#\#.\#";G1

IPRINT USING " HI=+\#\#\#\#.\#";H1

LPRINT

'Geographic colat. (TO) and lat1tude (T9) of North geomag. pole TO=FNRtOD $\left(-\operatorname{ATN}\left(\left(\mathrm{H}_{\left.\left.1-2+G 1-2)^{-} .5 / G O\right)\right)}\right.\right.\right.$ 'ATN is ArCTan $\mathrm{T} 9=90-\mathrm{TO}$

'West long. (LO) and east long. (L9) of North geomag. pole $L O=F N R$ tOD $(-\operatorname{ATN}(H 1 / G 1))$ 


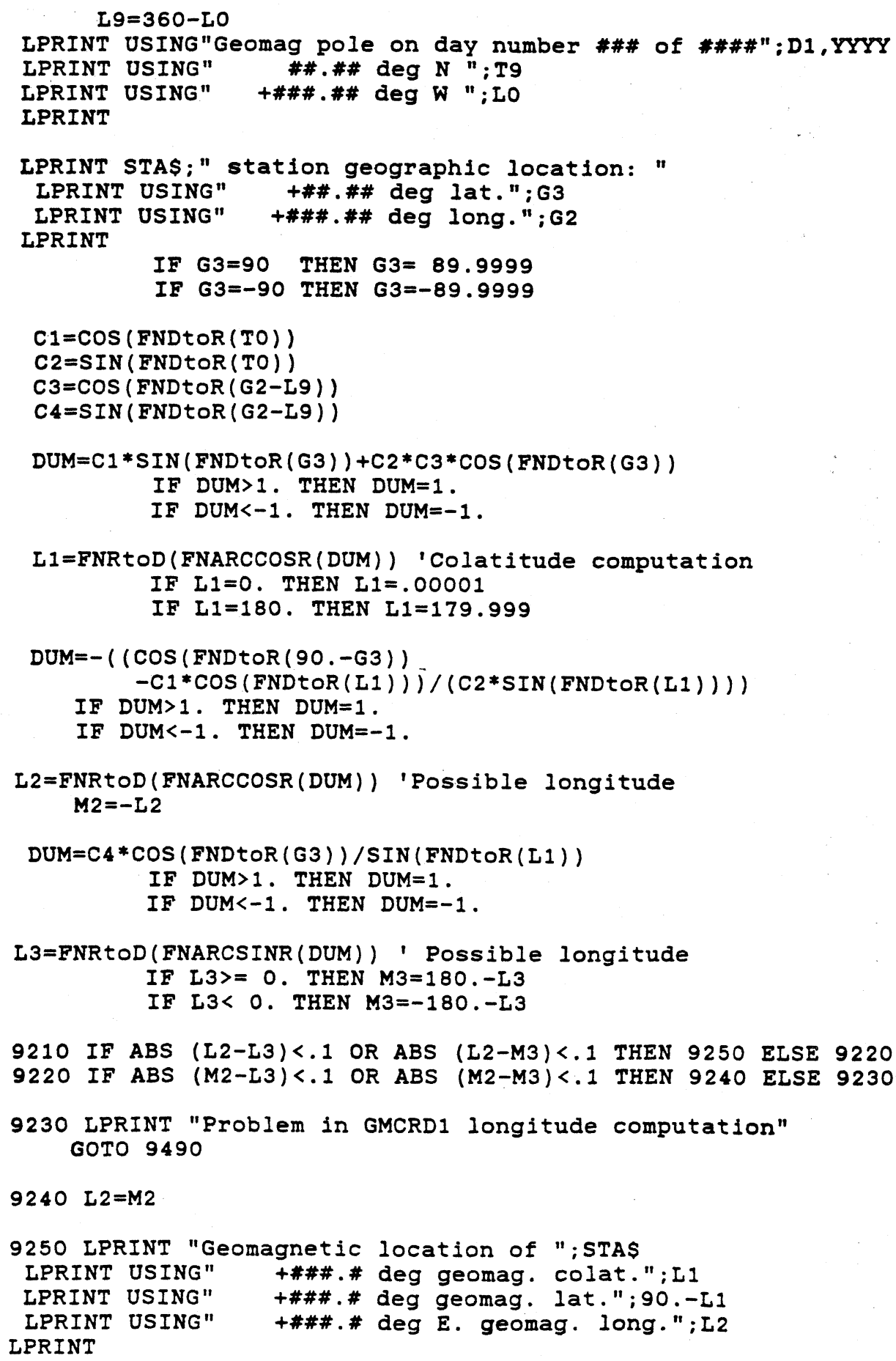




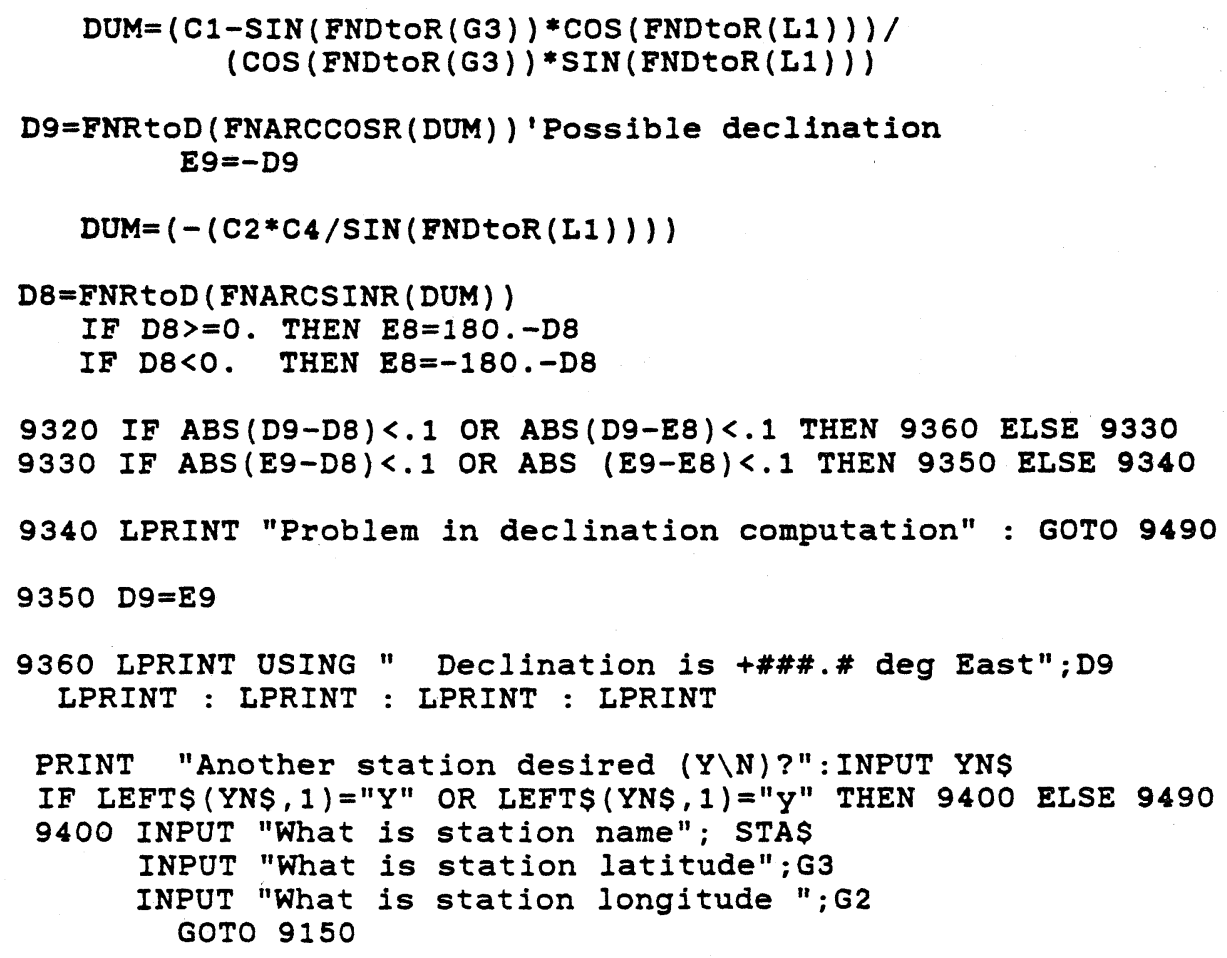


DATA $-30594.5,-2285,, 5809.5,-30554.1,-2250,, 5815.4,-30499.5$ DATA $-2215.4,5819.8,-30421 .,-2168.7,5791.4,-30334,,-2119$.

DATA $5776 \ldots,-30220 \ldots-2068 \ldots 5737 \ldots,-30100 \ldots-2013 \ldots, 5675$.

DATA $-29992,,-1956,5604,,-29877,,-1903,, 5497,,-29761$.

DATA $-1853 ., 5374.5,-29634, .-1795, .5199$.

'END OF DATA STATEMENTS 